\title{
Treatment Patterns Among Patients with Multiple Sclerosis Initiating Second-Line Disease-Modifying Therapy
}

\author{
James Bowen - Rina Mehta - Corey Pelletier - Marc Tian • \\ Virginia Noxon · Barbara H. Johnson · Machaon Bonafede
}

Received: March 13, 2020 / Published online: May 20, 2020

(C) The Author(s) 2020

\section{ABSTRACT}

Objective: Disease-modifying therapies (DMTs) can reduce multiple sclerosis (MS) relapse rates; however, effectiveness of treatments may vary. It is important to understand real-world treatment patterns in the context of MS relapses. We describe MS relapses related to treatment patterns among patients who switch treatment after their first DMT.

Methods: IBM MarketScan research databases were used to identify adult patients with MS who switched DMTs (index-first switch) after being newly treated with a DMT from January 2009 through March 2017, with 12 months of continuous enrollment pre- and post-index. Non-persistence was defined as discontinuing

Digital Features To view digital features for this article go to https://doi.org/10.6084/m9.figshare.12129501.

Electronic supplementary material The online version of this article (https://doi.org/10.1007/s12325020-01367-1) contains supplementary material, which is available to authorized users.

J. Bowen

Multiple Sclerosis Center, Swedish Neuroscience Institute, Seattle, WA, USA

R. Mehta · C. Pelletier · M. Tian

Bristol Myers Squibb, Summit, NJ, USA

V. Noxon $(\varangle) \cdot$ B. H. Johnson · M. Bonafede IBM Watson Health, Cambridge, MA, USA

e-mail: vnoxon@us.ibm.com (at least 60 days without DMT) or switching DMTs. MS relapses were defined using a validated claims-based algorithm. Multivariable analysis was used to examine odds of 12-month persistence, odds of post-index relapse, and number of relapses.

Results: In total, 4121 patients with MS met all inclusion criteria (mean age 46.4 years; female $76.2 \%$ ). Overall, $49.6 \%$ switched to an oral DMT, $36.5 \%$ to an injectable DMT, and $13.9 \%$ to an infusion DMT. Switching DMTs resulted in a $32.4 \%$ reduction in relapses between preand post-index. Only $54.6 \%$ of patients were persistent throughout the first year. Patients who switched to oral DMTs had 95\% higher adjusted odds of persistence and 18\% lower adjusted odds of a post-index period relapse than patients who switched to injectable DMTs. The number of baseline relapses was not associated with persistence but with $68 \%$ higher odds of a post-index relapse, with each additional baseline relapse associated with a $44 \%$ increase in number of post-index relapses.

Conclusions: Among patients with MS who switched DMTs, persistence was consistently low regardless of treatment. Although persistence with oral DMTs was slightly higher than with injectable DMTs, overall results indicate poor persistence to second-line therapy and highlight the need to improve long-term persistence with DMTs. 
Keywords: Adult; Drug therapy; Medication adherence; Retrospective studies; United States; Multiple sclerosis

\section{Key Summary Points}

Why carry out this study?

Multiple sclerosis is a chronic condition requiring a long-term management plan that responds to changes in disease status and patient characteristics

The disease-modifying treatment (DMT) options for multiple sclerosis have expanded rapidly in the past decade, but there is limited information to help guide treatment selection

This study examines treatment patterns and disease relapses among patients who switched DMTs

\section{What was learned from the study?}

During the 12-month observation period, $45.4 \%$ of patients were non-persistent, and patients who switched to oral DMTs had a higher odds of persistence and a lower odds of relapse than patients who switched to an injectable DMT after adjustment for baseline prognoses

Although persistence and relapse outcomes were better for patients who switched to an oral rather than an injectable DMT, the incidence of nonpersistence and relapse remained high, indicating a continuing need for improved treatments

\section{INTRODUCTION}

Multiple sclerosis (MS) is a chronic inflammatory condition characterized by immune-mediated attacks on the central nervous system [1], leading to demyelination along with axonal and neuronal injury [2]. The estimated prevalence of MS in the USA ranged from 337.9 to 362.2 per 100,000 people in 2017 , having climbed from 58 per 100,000 people in 1976 [3, 4]. MS is most prevalent among individuals aged 55-64 years [1] and is 2-3 times more common among women than men [4], with an age of onset typically between 20 and 40 years [1].

Current disease phenotypes include clinically isolated syndrome (CIS), relapsing-remitting MS (RRMS), progressive accumulation of disability from onset of primary progressive MS (PPMS), and progressive accumulation of disability after an initial relapsing course or secondary progressive MS (SPMS) [5]. CIS and RRMS may be further characterized as being active or not active; whereas, PPMS and SPMS are characterized by both disease activity and by whether the disease is with or without progression [5]. Disease severity and phenotype are determined through the evaluation of clinical characteristics (demographics, relapse severity, type of attack, relapse frequency, and disease course) and magnetic resonance imaging (MRI) features at onset and at follow-up [6].

Although there is no cure for MS, early control of symptoms and disease progression is associated with delayed disability and improved long-term outcomes [7, 8]. Disease-modifying therapies (DMTs) are the mainstay of MS treatment and can be delivered through three routes of administration (oral, injectable, and infusion) $[2,6,8,9]$. Mechanism of action and pharmacodynamics influence the efficacy and safety of an individual DMT [10]. Treatment with a DMT should be initiated as soon as possible following a diagnosis of CIS or RRMS or following a clinical relapse or an MRI showing inflammatory activity in patients who delayed early treatment [8]. The risk of imminent progression should guide the choice of treatment [6]. An escalation approach to therapy, which begins with relatively safer agents, should be used for most patients, and treatment switches should be considered after breakthrough disease [11]. Induction therapy, which uses high-efficacy treatment over the short term to obtain rapid control, is recommended for patients with aggressive disease or indicators of poor prognosis $[6,11]$. 
Once disease is controlled, the DMT should be continued indefinitely unless the patient has evidence of breakthrough disease activity (relapses or MRI lesions), intolerable side effects, inadequate adherence to medication, or access to a more appropriate treatment option [9]. Because MS is a progressive, lifelong condition, patients commonly switch therapies over the course of their disease [10]. In previous studies, $19-28 \%$ of patients switched therapies at least once within 2 years of initiating treatment with a DMT $[12,13]$.

The reason for needing to switch often drives the selection of the subsequent DMT; however, route of administration may influence treatment decisions $[13,14]$. Treatment sequencing should consider the mechanism of action of both treatments and the potential impact of the initial therapy on the efficacy and toxicity of the subsequent therapy [6]. For example, switching from medications with long-lasting immunosuppressive effects (ocrelizumab or alemtuzumab) to natalizumab may require the use of a washout period or bridging medication to reduce the risk of progressive multifocal leukoencephalopathy [15].

This study updates existing real-world evidence and research by describing treatment patterns and treatment switching across all MS DMTs available to US patients between January 1, 2009, and April 1, 2017. This study used administrative claims data to describe treatment patterns in patients initiating second-line DMTs (first switch). Treatment patterns and switching were also assessed by route of administration.

\section{METHODS}

\section{Study Design and Data Source}

This retrospective, administrative claims-based study analyzed treatment patterns and relapses among commercially insured adults who switched to a second DMT for MS in the USA. Deidentified longitudinal patient data were extracted from the IBM MarketScan Commercial Claims and Encounters Database and the IBM MarketScan Medicare Supplemental and Coordination of Benefits Database (IBM Watson
Health, Cambridge, MA) from January 1, 2009, through March 31, 2018 (study period). Each database captures the inpatient medical, outpatient medical, and outpatient prescription drug data for its respective covered population, and together, they form a nationally representative, convenience sample of insured individuals living in the USA.

All study data were obtained using International Classification of Diseases, Ninth and Tenth Revisions, Clinical Modification codes (ICD-9-CM and ICD-10-CM), Current Procedural Terminology Fourth Edition codes, Healthcare Common Procedure Coding System (HCPCS) codes, and National Drug Codes (NDCs).

All database records were statistically deidentified and certified as fully compliant with US patient confidentiality requirements set forth in the Health Insurance Portability and Accountability Act of 1996. Because this study used only de-identified patient records and did not involve the collection, use, or transmittal of individually identifiable data, institutional review board approval was not necessary.

\section{Patient Selection and Cohort Assignment}

Patients with a diagnosis of MS and at least two nondiagnostic claims (at least 1 day and less than 365 days apart) between January 1, 2009, and April 1, 2017, were identified in the MarketScan commercial and Medicare databases. Treatment-naïve patients were identified as those with at least one claim for a single MS DMT on or after the first MS claim and no claims for a DMT in the preceding 12 months. Eligible patients with MS also had to have switched to a second DMT from their initial DMT. The date of the DMT switch was set as the index date.

Patients who received more than one DMT on the index date were excluded, as were DMT users with evidence of pregnancy or primary malignancy anytime during the study. Patients who had switched DMTs were required to have had continuous health care enrollment in the 12 months prior to their first DMT and from the start of their first DMT to the index date and for 
at least 12 months before the index date (baseline period) and 12 months after the index date (follow-up period). Patients were stratified by route of administration (oral, injection, or infusion) of their index medication. This study included injectable (glatiramer acetate, interferon beta-1a [intramuscular and subcutaneous], interferon beta- $1 \mathrm{~b}$, and peginterferon beta-1a), oral (dimethyl fumarate, fingolimod, and teriflunomide), and infusion (natalizumab, alemtuzumab, and mitoxantrone) DMTs.

\section{Baseline Characteristics}

Baseline patient demographics on the index date were recorded and included age, sex, geographic region, index year, and time from the end of the first DMT to the index date. Baseline clinical characteristics during the 12-month baseline period were recorded and included the Deyo-Charlson Comorbidity Index [16], selected comorbid conditions (depression, hyperlipidemia, hypertension, fatigue, and neuropathic pain), and medications (antidepressants, antispasmodics, nonsteroidal antiinflammatory drugs [NSAIDs] or cyclooxygenase-2 [COX-2] inhibitors, neuropathic pain medications, and opioids).

\section{Relapses}

Relapses occurring in the baseline and followup periods were identified using the following validated administrative claims-based algorithm [17]: (1) any inpatient stay with an MS diagnosis in the primary position or (2) any outpatient claim with an MS diagnosis code in the primary or secondary position followed within 7 days by a pharmacy or medical claim for adrenocorticotropin hormone, dexamethasone, methylprednisolone, prednisolone, or prednisone. The number of patients with one, two, and three or more relapses and the mean number of relapses per DMT user were recorded.

\section{Treatment Patterns}

Adherence to the index DMT in the follow-up period was calculated as the percentage of days covered (PDC). Overlapping days' supply between consecutive claims was appended for medications identified by NDCs and truncated for medications identified by HCPCS. DMT users with a PDC of at least $80 \%$ were considered adherent.

Non-persistence to the index DMT was determined in the follow-up period and was caused by study discontinuation, which was defined as a gap of at least 60 days after the end of days' supply of the index DMT or a switch to a new DMT. Days' supply was determined by the number of days of supply of medications identified by NDCs or by dosing schedule for medications identified by HCPCS. Treatment persistence was measured at 3,6 , and 12 months post-index.

\section{Statistical Analyses}

Descriptive statistics were used to report baseline characteristics and study outcomes. Continuous variables were reported as mean and standard deviation. Categorical variables were reported as the count and percentage of patients.

Multivariable models were used to examine persistence and relapse outcomes while adjusting for baseline differences between patient subgroups. Logistic regression was used to examine the odds of persistence with treatment at 12 months after DMT switch and the odds of relapse within 12 months after DMT switch. Poisson regression was used to examine the number of relapses observed within 12 months after switching DMTs. Route of administration of the index DMT was the independent variable of interest in all models, with injectable initiators as the reference group. All models adjusted for select patient characteristics that included the initial DMT route of administration, time from end of initial DMT to index date, number of baseline relapses, age (by decade), sex, index year (categorical for each year), geographic region of residence, select comorbid conditions (depression, hyperlipidemia, hypertension, fatigue, and neuropathic pain), and baseline medications (antidepressants, antispasmodics, NSAIDs or COX-2 inhibitors, neuropathic pain 
medications, and opioids). Results of logistic regression are reported as odds ratios (ORs) with 95\% confidence intervals (CIs), while the results of Poisson regression are reported as count ratios (CRs) with 95\% CIs.

A $P$ value of less than 0.05 was set a priori as the threshold for statistical significance. All analyses were conducted using WPS version 4.1 (World Programming, UK).

\section{RESULTS}

\section{Patient Characteristics}

Of the 227,893 identified patients with MS, 25,708 (11.3\%) were considered DMT naïve and, among these, 4121 (16.0\%) switched to a second discrete DMT and met the final inclusion criteria. The full patient selection can be found in Fig. 1. Patients generally switched to an oral $(49.6 \%)$ or injectable $(36.5 \%)$ DMT. Of the $13.9 \%$ of patients who switched to an infusion, 94\% switched to natalizumab.

The majority of patients who switched were receiving an injectable DMT. Of those who switched (indexed on) to an oral, injectable, or infusion, $80.5 \%, 82.9 \%$, and $80.6 \%$ did so from an injectable (Table $S 1$ in the supplementary material). Patients generally switched within 5 months after discontinuing their first DMT (Table 1), and the mean time from the end of the initial DMT to the switch ranged from 110.3 days for switching to injectable DMTs to 169.6 days for switching to oral DMTs.

Patient demographics were consistent across routes of administration, except for during the index years, which were driven by US Food and Drug Administration approval of specific drugs. Patients who switched DMTs were, on average, 46.4 years of age at the time of the switch (Table 1). The majority of patients were female (76.2\%), from a southern geographic region (38.8\%), and switched their DMT in 2013 or later $(64.1 \%)$.

Comorbid conditions and concomitant medication use were consistent across all routes of administration and among those whose first DMT was injectable. Among the comorbid conditions examined, fatigue (24.5\%) and hypertension $(21.6 \%)$ were the most commonly observed conditions before patients switched DMTs (Table 1). Among medication classes examined, opioids (41.5\%) and antidepressants (39.9\%) were the most commonly used drugs before patients switched DMTs.

\section{Treatment Patterns}

More than $40 \%$ of all patients were non-persistent with index treatment in a 12-month period; those who initiated injectables as a secondline treatment (i.e., index treatment; switch to) had the highest proportion of patients (54.5\%) who were non-persistent (Table 2). Among patients who were non-persistent, most $(88.2 \%)$ discontinued their DMT. Restarting the index DMT after discontinuing was uncommon $(6.7 \%)$ but generally occurred within 110 days after discontinuation.

Roughly half $(49.5 \%)$ of patients were nonadherent to the switched treatment within 1 year of initiation (Table 2). The largest percentage of adherent patients switched to infusion DMTs $(62.1 \%)$, followed by oral $(53.0 \%)$ and injectable DMTs (42.8\%). The mean PDC for all patients who switched DMTs was 0.66.

Treatment adherence and persistence were consistent across all routes of administration, but patients who switched to oral DMTs were more adherent and persistent than patients who switched to injectable DMTs, especially those starting with an injectable. Among patients initially receiving injectable DMTs, those who switched to oral DMTs were more adherent (55.0\%) and persistent (60.4\%) than those who switched to another injectable $(43.2 \%$ and $46.2 \%$, respectively) (Table 22 in the supplementary material). Among patients initially receiving oral DMTs, those who switched to another oral DMT were more likely to be persistent $(44.3 \%)$ than those who switched to an injectable DMT (43.5\%) (Table 3).

\section{Relapses}

Among patients with MS who switched medications, there was a 14.8 percentage point increase in the number of patients without a 


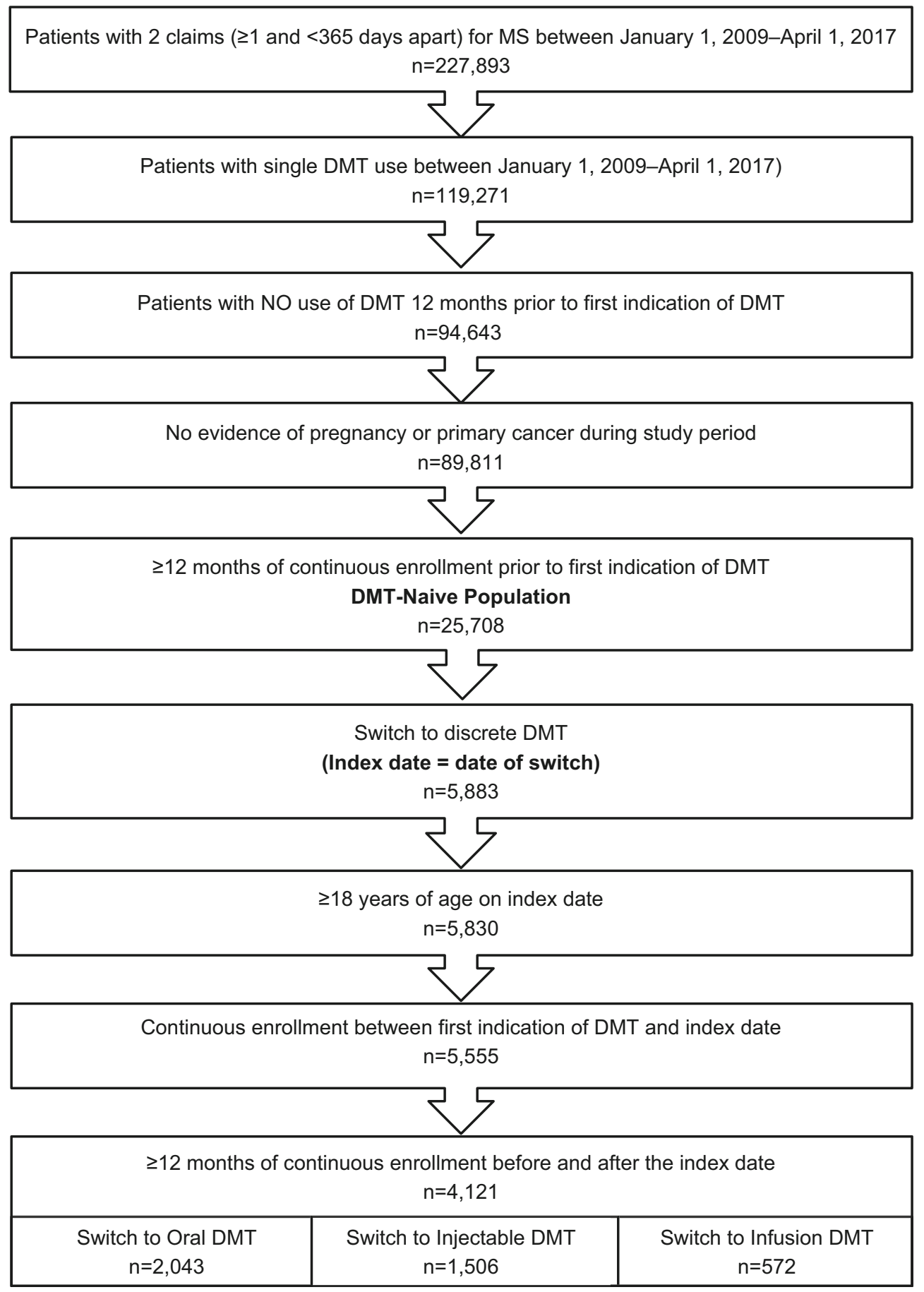

Fig. 1 Patient selection. DMT disease-modifying therapy, MS multiple sclerosis

relapse between baseline and follow-up (Fig. 2). After switching DMTs, $75.4 \%$ of those who switched to oral DMTs were relapse-free compared with $70.4 \%$ and $60.7 \%$ of those who switched to injectable and infusion DMTs, respectively.

Among patients whose initial DMTs were injectable, those who switched to oral DMTs 
Table 1 Patient characteristics among patients switching disease-modifying therapies

\begin{tabular}{|c|c|c|c|c|}
\hline Parameter & $\begin{array}{l}\text { All patients } \\
(n=4121)\end{array}$ & $\begin{array}{l}\text { Oral DMT patients } \\
(n=2043)\end{array}$ & $\begin{array}{l}\text { Injectable DMT } \\
\text { patients }(n=1506)\end{array}$ & $\begin{array}{l}\text { Infusion DMT } \\
\text { patients }(n=572)\end{array}$ \\
\hline Age, mean (SD), years & $46.4(10.9)$ & $47.1(10.6)$ & $46.2(11.0)$ & $44.2(11.5)$ \\
\hline Female $[n(\%)]$ & $3140(76.2)$ & $1523(74.5)$ & $1185(78.7)$ & $432(75.5)$ \\
\hline \multicolumn{5}{|l|}{ Geographic region $[n(\%)]$} \\
\hline Northeast & $759(18.4)$ & $378(18.5)$ & $275(18.3)$ & $106(18.5)$ \\
\hline North central & $997(24.2)$ & $523(25.6)$ & $369(24.5)$ & $105(18.4)$ \\
\hline South & $1599(38.8)$ & $771(37.7)$ & $556(36.9)$ & $272(47.6)$ \\
\hline West & $737(17.9)$ & $355(17.4)$ & $296(19.7)$ & $86(15.0)$ \\
\hline Unknown & $29(0.7)$ & $16(0.8)$ & $10(0.7)$ & $3(0.5)$ \\
\hline \multicolumn{5}{|l|}{ Index year $[n(\%)]$} \\
\hline 2009 & $158(3.8)$ & $0(0.0)$ & $141(9.4)$ & $17(3.0)$ \\
\hline 2010 & $298(7.2)$ & $16(0.8)$ & $225(14.9)$ & $57(10.0)$ \\
\hline 2011 & $549(13.3)$ & $162(7.9)$ & $289(19.2)$ & $98(17.1)$ \\
\hline 2012 & $476(11.6)$ & $123(6.0)$ & $252(16.7)$ & $101(17.7)$ \\
\hline 2013 & $917(22.3)$ & $703(34.4)$ & $139(9.2)$ & $75(13.1)$ \\
\hline 2014 & $566(13.7)$ & $395(19.3)$ & $119(7.9)$ & $52(9.1)$ \\
\hline 2015 & $563(13.7)$ & $310(15.2)$ & $179(11.9)$ & $74(12.9)$ \\
\hline 2016 & $491(11.9)$ & $281(13.8)$ & $133(8.8)$ & $77(13.5)$ \\
\hline 2017 & $103(2.5)$ & $53(2.6)$ & $29(1.9)$ & $21(3.7)$ \\
\hline $\begin{array}{l}\text { Days from first DMT to } \\
\text { switch, }{ }^{a} \text { mean }(\mathrm{SD})\end{array}$ & $140.8(297.9)$ & $169.6(330.5)$ & $110.3(267.0)$ & $118.5(236.6)$ \\
\hline \multicolumn{5}{|c|}{ Comorbid conditions $[n(\%)]$} \\
\hline Fatigue & $1009(24.5)$ & $487(23.8)$ & $362(24.0)$ & $160(28.0)$ \\
\hline Hypertension & $892(21.6)$ & $450(22.0)$ & $331(22.0)$ & $111(19.4)$ \\
\hline Depression & $805(19.5)$ & $372(18.2)$ & $298(19.8)$ & $135(23.6)$ \\
\hline Gait difficulties & $647(15.7)$ & $292(14.3)$ & $221(14.7)$ & $134(23.4)$ \\
\hline Hyperlipidemia & $625(15.2)$ & $323(15.8)$ & $227(15.1)$ & $75(13.1)$ \\
\hline \multicolumn{5}{|c|}{ Concomitant medications $[n(\%)]$} \\
\hline Opioids & $1711(41.5)$ & $809(39.6)$ & $671(44.6)$ & $231(40.4)$ \\
\hline Antidepressants & $1646(39.9)$ & $794(38.9)$ & $607(40.3)$ & $245(42.8)$ \\
\hline Antispasmodics & $1460(35.4)$ & $700(34.3)$ & $508(33.7)$ & $252(44.1)$ \\
\hline $\begin{array}{l}\text { Neuropathic pain } \\
\text { medications }\end{array}$ & $1318(32.0)$ & $631(30.9)$ & $484(32.1)$ & $203(35.5)$ \\
\hline $\begin{array}{l}\text { NSAIDs/COX-2 } \\
\text { inhibitors }\end{array}$ & $1177(28.6)$ & $563(27.6)$ & $437(29.0)$ & $177(30.9)$ \\
\hline
\end{tabular}

${ }^{a}$ From end of days supply or clinical benefit of last claim for first DMT until index date

COX-2 cyclooxygenase-2, DMT disease-modifying therapy, NSAIDs nonsteroidal anti-inflammatory drugs 
Table 2 Treatment patterns among patients switching disease-modifying therapies

\begin{tabular}{|c|c|c|c|c|}
\hline Parameter & $\begin{array}{l}\text { All patients } \\
(n=4121)\end{array}$ & $\begin{array}{l}\text { Oral DMT patients } \\
(n=2043)\end{array}$ & $\begin{array}{l}\text { Injectable DMT } \\
\text { patients }(n=1506)\end{array}$ & $\begin{array}{l}\text { Infusion DMT } \\
\text { patients }(n=572)\end{array}$ \\
\hline \multicolumn{5}{|l|}{ Persistence } \\
\hline $\begin{array}{l}\text { Persistent patients } \\
{[n(\%)]}\end{array}$ & $2250(54.6)$ & $1190(58.2)$ & $685(45.5)$ & $375(65.6)$ \\
\hline $\begin{array}{l}\text { Non-persistent patients } \\
{[n(\%)]}\end{array}$ & $1871(45.4)$ & $853(41.8)$ & $821(54.5)$ & $197(34.4)$ \\
\hline Due to discontinuation & $1651(88.2)$ & $765(89.7)$ & $706(86.0)$ & $180(91.4)$ \\
\hline Due to switch & $220(11.8)$ & $88(10.3)$ & $115(14.0)$ & $17(8.6)$ \\
\hline $\begin{array}{l}\text { Time to non-persistence, } \\
\text { mean (SD) }\end{array}$ & $128.8(90.3)$ & $123.7(88.6)$ & $134.3(91.5)$ & $128.4(91.6)$ \\
\hline $\begin{array}{l}\text { Time on treatment in } \\
\text { days, mean }(\mathrm{SD})\end{array}$ & $255.9(131.1)$ & $262.3(130.8)$ & $237.5(131.9)$ & $281.4(123.6)$ \\
\hline \multicolumn{5}{|l|}{ Adherence } \\
\hline PDC, mean $(S D)$ & $0.66(0.32)$ & $0.68(0.31)$ & $0.62(0.33)$ & $0.74(0.29)$ \\
\hline $\begin{array}{l}\text { Adherent patients } \\
{[n(\%)]}\end{array}$ & $2082(50.5)$ & $1083(53.0)$ & $644(42.8)$ & $355(62.1)$ \\
\hline \multicolumn{5}{|l|}{ Restart index DMT } \\
\hline $\begin{array}{l}\text { Restarted the index } \\
\text { DMT }[n(\%)]\end{array}$ & $278(6.7)$ & $134(6.6)$ & $112(7.4)$ & $32(5.6)$ \\
\hline $\begin{array}{l}\text { Time to restart, mean } \\
\text { (SD) }\end{array}$ & $109.5(57.2)$ & $107.1(55.4)$ & $104.5(56.3)$ & $136.9(62.2)$ \\
\hline
\end{tabular}

$D M T$ disease-modifying therapy, $P D C$ percentage of days covered

were less likely to experience a relapse $(22.4 \%)$ than those who switched to another injectable DMT (28.5\%) (Table 3). The number of observed relapses among patients starting therapy with injectable DMTs was also lower among those who switched to oral DMTs (0.36) than among those who switched to another injectable (0.47) (Table 3). A similar trend was observed among patients switching from an initial oral to another oral DMT (0.42) compared with those switching from an oral to an injectable DMT (0.48).

Patients who switched to oral DMTs had greater reductions in the number of those with at least three follow-up relapses $(28.6 \%$ reduction vs baseline) compared with patients who switched to injectable DMTs (6.3\% reduction)
(Fig. 2). Patients who started on injectable DMTs showed a similar trend in the reduction in the number of patients with at least three follow-up relapses $(24.2 \%$ switching to oral and $3.4 \%$ for switching to injectable DMTs) (Fig. S1 in the supplementary material). The more relapses a patient experienced before starting a DMT, the more likely that patient was to experience a relapse after starting the DMT (Fig. 3). More than 60\% of patients switching to oral and infusion DMTs and more than half of those switching to injectable DMTs (56.2\%) who had at least three relapses in the baseline period had from zero to one relapse in the first year after switching (Fig. S2 in the supplementary material). 
Table 3 Persistence and relapses by DMT switch sequence

\begin{tabular}{lcclll}
\hline First DMT & Index DMT & Patients $(\boldsymbol{n})$ & $\begin{array}{l}\text { Persistent } \\
\text { patients (\%) }\end{array}$ & $\begin{array}{l}\text { Patients with } \\
\text { relapse after } \\
\text { switch (\%) }\end{array}$ & $\begin{array}{l}\text { No. of } \\
\text { relapses after } \\
\text { switch }\end{array}$ \\
\hline All & a & $\mathbf{5 7 2}$ & $\mathbf{6 5 . 6}$ & $\mathbf{3 9 . 3}$ & $\mathbf{0 . 6 8}$ \\
Infusion & & 15 & 100.0 & 80.0 & 1.00 \\
Injectable & Infusion & 461 & 68.8 & 38.0 & 0.65 \\
Oral & & 46.8 & 39.6 & 0.78 \\
All & & $\mathbf{1 5 0 6}$ & $\mathbf{4 5 . 5}$ & $\mathbf{2 9 . 6}$ & $\mathbf{0 . 4 8}$ \\
Infusion & & 38.9 & 41.7 & 0.69 \\
Injectable & Injectable & 1248 & 46.2 & 28.5 & 0.47 \\
Oral & 186 & 43.5 & 32.3 & 0.48 \\
All & $\mathbf{2 0 4 3}$ & $\mathbf{5 8 . 2}$ & $\mathbf{2 4 . 6}$ & $\mathbf{0 . 3 9}$ \\
Infusion & 168 & 56.0 & 35.1 & 0.60 \\
Injectable & Oral & 1645 & 60.4 & 22.4 & 0.36 \\
Oral & & 44.3 & 33.0 & 0.42 \\
\hline
\end{tabular}

$D M T$ disease-modifying therapy

a All patients with an infusion as a first and second DMT had alemtuzumab as their second DMT (index treatment) which has a 1-year clinical benefit so are automatically considered persistent

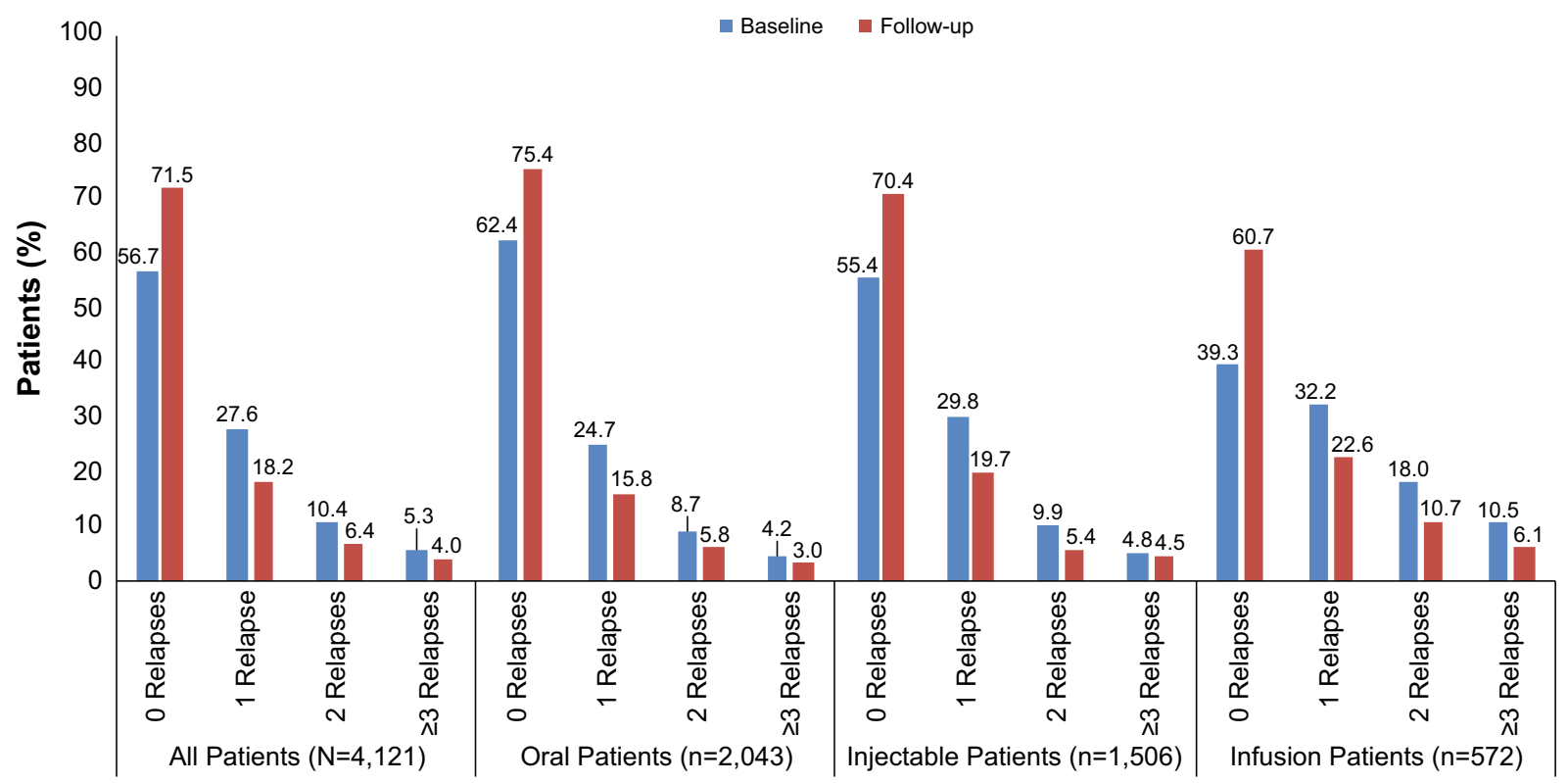

Fig. 2 Patients with at least 1 relapse during the baseline and follow-up periods in patients who switched disease-modifying therapies 


\section{Relapses and Persistence}

Relapses before switching DMTs did not appear to predict DMT persistence within the first year of the switch (Fig. S3 in the supplementary material). However, relapses within the first 3 months of switching correlated with lower persistence at 6 and 12 months after the DMT switch (Fig. 4). Relapses within the first 6 months of switching also correlated with lower DMT persistence at 12 months. Compared with patients who switched to injectable DMTs, those who switched to oral DMTs were more likely to be persistent at 12 months, regardless of whether they experienced a relapse in the first 3 months (Fig. S4 in the supplementary material).

\section{Multivariate Results}

Patients who switched to oral DMTs were 95\% more likely $(P<0.0001)$ to be persistent at 12 months than those who switched to injectable DMTs after adjusting for patient characteristics (Fig. 5, Table S3 in the supplementary material). Patients switching to infusion DMTs were $195 \%$ more likely $(P<0.0001)$ to be persistent at 12 months than those switching to injectable DMTs. The number of baseline relapses was not significantly associated with persistence at 12 months $(P=0.9995)$. A larger gap between the end of first DMT and the start of the second DMT was significantly associated with non-persistence (91-180 days OR [95\% CI] 0.75 [0.58-0.96], $P<0.05 ;>180$ days OR $[95 \%$ CI $] \quad 0.61$ [0.50-0.74], $P<0.0001)$.

Patients switching to oral DMTs were $18 \%$ less likely $(P<0.05)$ to experience a relapse within 12 months after switching compared with those switching to injectable DMTs, after adjusting for patient characteristics (Fig. 5, Table S4 in the supplementary material). Switching to an infusion DMT did not have a significant association with experiencing a relapse within 12 months $(P=0.1126)$. Among all patients, each additional relapse observed prior to the DMT switch was associated with a $68 \%$ increase in odds of a relapse after DMT initiation $(P<0.0001)$.

Patients who switched to oral DMTs were less likely to experience more relapses in the 12 months after switching compared with those who switched to injectable DMTs (CR [95\% CI] 0.84 [0.75-0.94], $P<0.01$ ) (Fig. 5, Table S5 in the supplementary material). Patients who switched to a new infusion DMT (regardless of

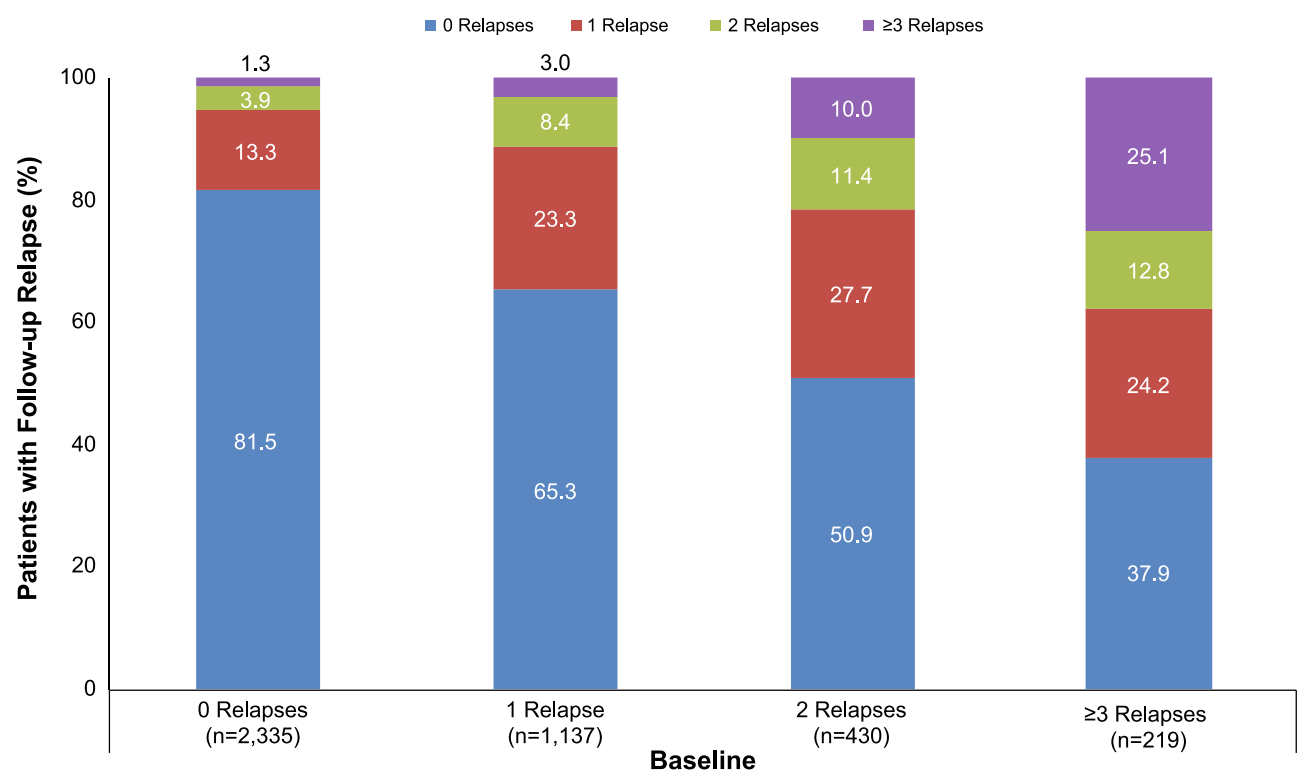

Fig. 3 Association between baseline relapses and follow-up relapses among all patients. Describes patients who have 0, 1, 2, or $\geq 3$ relapses in the baseline period who had $0,1,2$, or $\geq 3$ relapses in the follow-up period 


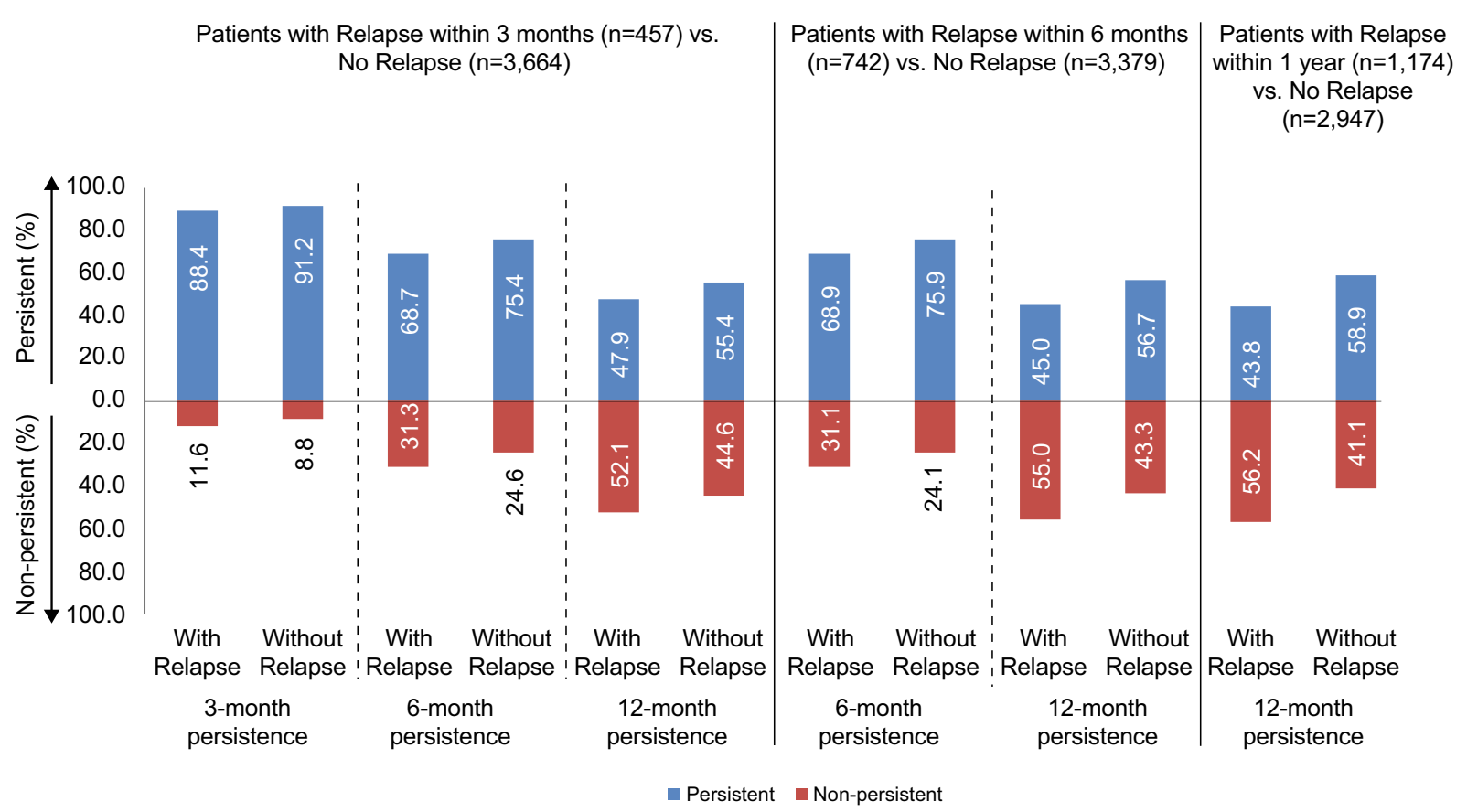

Fig. 4 Association between relapses after disease-modifying therapy initiation and disease-modifying therapy persistence at 3, 6, and 12 months. Describes patients who do and do not have a relapse during follow-up and those who are persistent and not persistent in each cohort

first DMT) were more likely to have an increase in the number of relapses experienced within 12 months (CR [95\% CI] 1.18 [1.04-1.34], $P<0.05)$. Among all patients, each additional relapse observed prior to DMT initiation was associated with a $44 \%$ increase in the number of relapses after DMT initiation $(P<0.0001)$.

\section{DISCUSSION}

This real-world analysis examined treatment patterns, relapses, and the association between relapses and treatment persistence among patients with MS who switched DMTs and adds to the limited literature describing the impact of switching from an initial to a second DMT. Among patients who switched DMTs, oral agents were the most common second therapy, a finding that is consistent with that of prior studies. A study of Medicare claims showed that patients more commonly switched to oral (relapse, non-relapse). Relapses were determined at 3, 6, and 12 months during follow-up. Persistence was determined at time of relapse and for longer periods in followup

DMTs (more than 80\%) over injectables and infusions [18]. Although this rate is much higher than in our results $(49.6 \%)$, use of the most recent prescribing patterns versus the earliest patterns and limiting the study population to Medicare patients preclude a direct comparison between studies. A second claims study showed that oral agents may be the new preferred second DMT treatment, as $6 \%$ of patients who switched in the second half of 2010 and $79 \%$ of those who switched in the second half of 2014 were treated orally after the switch [19]. Although their data show a larger proportion of patients switching to oral DMTs, these investigators likely included first as well as second or third switches and we report only the first switch [19]. In our analysis, patients with MS showed a reduction in relapses after switching to a new DMT, with the number of relapse-free patients increasing 15 percentage points in the year following treatment switching. Despite switching treatments, $29 \%$ of patients experienced at least one relapse during 


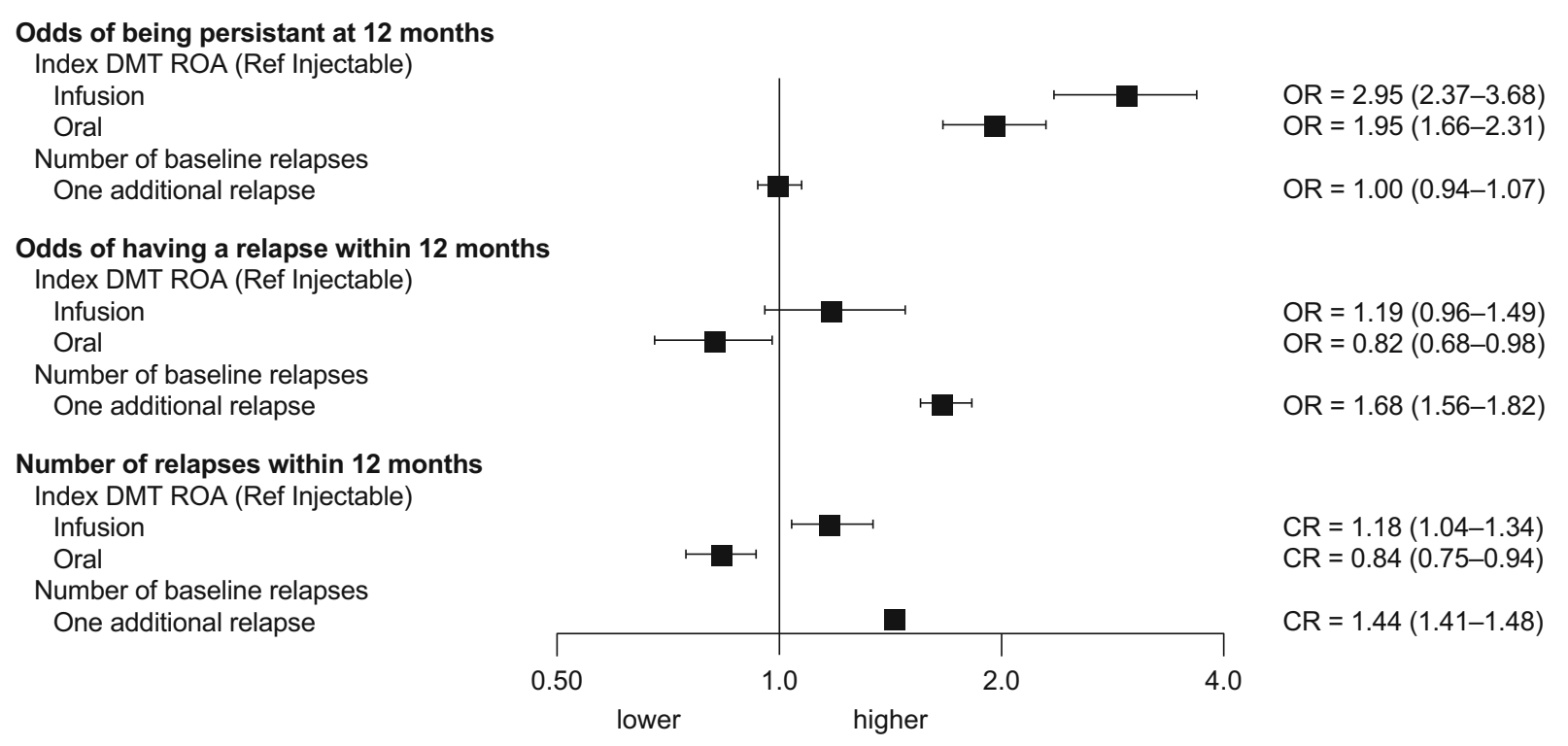

Fig. 5 Multivariate models ${ }^{a}$ : odds of persistence at 12 months ${ }^{\mathrm{b}}$, odds of relapse within 12 months $^{\mathrm{b}}$, and count of additional relapses within 12 months ${ }^{c}$. ${ }^{a}$ Models adjusted for first disease-modifying therapy route of administration, time from end of first DMT to index date, age, sex, index year, geographic region, prior indication of fatigue, neuropathic pain, hypertension,

the follow-up period and prior relapses increased the odds and number of relapses in the follow-up period.

Forty-eight percent of patients who switched to an oral DMT experienced at least one relapse during the baseline period compared with $45 \%$ of patients who switched to an injectable DMT and $61 \%$ of patients who switched to an infusion DMT. This suggests that patients who switched to oral DMTs may be doing so for reasons other than the failure of their first-line medication, whereas patients who switched to an infusion DMT were more likely to be doing so because of prior treatment failure. However, even after adjusting for the number of baseline relapses, switching to an oral DMT was associated with reduced odds of having a relapse in the follow-up period and a reduced number of relapses compared with switching to an injectable DMT.

One-year persistence and adherence were roughly 50\% for patients who switched DMTs. Persistence and adherence were highest for hyperlipidemia, or depression, and prior use of antidepressants, opioids, antispasmodics, neuropathic pain treatments, or NSAID/COX-2 inhibitors. ${ }^{b}$ Logistic regression model. ${ }^{\mathrm{c}}$ Poisson regression model. COX-2 cyclooxygenase2, CR count ratio, NSAIDs nonsteroidal anti-inflammatory drugs, $\mathrm{ROA}$ route of administration

those who switched to an infusion DMT and lowest for those who switched to an injectable DMT. After adjustment for baseline characteristics, the odds of being persistent at 12 months were higher for patients who switched to an oral or infusion DMT compared with those who switched to an injectable DMT. Across all patients who switched DMTs, persistence dropped from $91 \%$ at 3 months to $75 \%$ at 6 months and to $55 \%$ at 12 months. Patients who experienced a relapse were less likely to be persistent, but there did not appear to be a correlation between the timing of the relapse and non-persistence.

As new treatments have become available, treatment patterns among patients with MS have shifted. A prior administrative claims study spanning 2007 through 2011 found that among the 1095 patients with MS who started on an injectable DMT and switched therapies within 2 years, $79 \%$ switched to a different injectable, $19 \%$ switched to an infusion DMT, and 2\% switched to an oral DMT [12]. A chart review study 
spanning 2008 through 2015 found that of the 84 patients who started on an injectable DMT and switched treatments within 2 years, 37\% switched to a different injectable, $30 \%$ switched to an infusion DMT, and 33\% switched to an oral DMT [13]. By comparison, in this study spanning 2008 through 2018, of the 3354 patients who switched from an injectable DMT, 37\% switched to a different injectable and $14 \%$ switched to an infusion DMT, while $49 \%$ switched to an oral DMT. In a study of German health records spanning 2010 through 2015, patients who switched from an injectable DMT because of treatment failure to orally administered fingolimod had a lower risk of discontinuation and a lower risk of relapse than patients who switched to a different injectable DMT [20].

\section{Limitations}

The limitations of this study are those inherent in any retrospective analysis. First, the study was limited to only those individuals with commercial health coverage or private Medicare supplemental coverage. Consequently, the results of this analysis may not be generalizable to patients with MS who have other insurance or are without health insurance coverage. Second, the potential for misclassification of MS status, clinical and patient demographics and characteristics, as well as study outcomes (i.e., MS relapse) were present, as patients were identified through administrative claims data as opposed to medical records. As with any claims databases, the MarketScan research databases rely on administrative claims data for clinical detail. As such, the specific type of MS was unable to be determined so any differences in treatment due to type of MS were unable to be evaluated. These data are subject to data coding limitations and data entry errors. Third, medication compliance and persistence were based on filled prescriptions. It was assumed that patients took the medications as prescribed; however, we could not confirm how patients actually took the medications. Additionally, we were not able to capture medications administered in the inpatient or emergency department setting in the Commercial and Medicare Supplemental MarketScan databases. Fourth, the current study included a 12-month follow-up period. Patients who died or who went on long-term disability due to serious health conditions had a follow-up period of less than 12 months and, therefore, were excluded from the analysis. Lastly, the reason for discontinuation cannot be determined from claims data; therefore, this analysis cannot distinguish between patients who became non-persistent because of lack of efficacy, side effects, or other reasons.

\section{CONCLUSIONS}

Treatment-naïve patients who need to switch medications may have better outcomes if switched to an oral compared with an injectable DMT, as oral DMT usage may be associated with longer adherence and persistence. Although improvements in the number of relapses were observed after switching to a second DMT (regardless of route of administration), and these improvements were better among patients who switched to oral versus another injectable, nearly $30 \%$ of patients who switched medications had at least one relapse in the following year. This underscores a continued need for switching to medications with greater efficacy that offer a better benefit-to-risk ratio profile, reduced relapses, and greater safety and tolerability.

\section{ACKNOWLEDGEMENTS}

Funding. This study, including the Rapid Service Fee and the Open Access Fee, was sponsored by Celgene, a Bristol Myers Squibb company.

Medical Writing, Editorial, and other Assistance. The authors received medical writing support from Jessamine Winer-Jones of IBM Watson Health, Cambridge, MA, USA, and editorial support from Peloton Advantage, LLC, an OPEN Health company, Parsippany, NJ, USA, sponsored by Bristol Myers Squibb, Summit, NJ, USA. The authors, however, directed and are 
fully responsible for all content and editorial decisions for this manuscript.

Authorship. All named authors meet the International Committee of Medical Journal Editors (ICMJE) criteria for authorship for this article, take responsibility for the integrity of the work as a whole, and have given their approval for this version to be published.

Disclosures. James Bowen is a paid consultant of Bristol Myers Squibb, Summit, NJ, USA. Corey Pelletier is employed by Bristol Myers Squibb. Rina Mehta and Marc Tian were employed by Celgene, now a wholly-owned subsidiary of Bristol Myers Squibb, at the time the study was conducted. Virginia Noxon is employed by IBM Watson Health, which received funding from Celgene, now a wholly-owned subsidiary of Bristol Myers Squibb, to conduct this study. Machaon Bonafede and Barbara $\mathrm{H}$. Johnson were employed by IBM Watson Health at the time the study was conducted. Rina Mehta works at Novartis Pharmaceuticals in East Hanover, NJ, USA; Marc Tian works at Teva Pharmaceutical Industries, West Chester, PA, USA; Machaon Bonafede works at Veradigm, an Allscripts company, in Chicago, IL, USA; and Barbara Johnson works at Johnson \& Johnson in New Brunswick, NJ, USA.

Prior Presentation. Portions of this research are scheduled to be available for the virtual meeting of the 2020 International Society for Pharmacoeconomics and Outcomes Research, May 18-20, 2020.

Compliance with Ethics Guidelines. All database records were statistically de-identified and certified as fully compliant with US patient confidentiality requirements set forth in the Health Insurance Portability and Accountability Act of 1996. Because this study used only deidentified patient records and did not involve the collection, use, or transmittal of individually identifiable data, institutional review board approval was not necessary.

Data Availability. The datasets generated during and analyzed for the current study are not publicly available as they were used under license from IBM Watson Health.

Open Access. This article is licensed under a Creative Commons Attribution-NonCommercial 4.0 International License, which permits any non-commercial use, sharing, adaptation, distribution and reproduction in any medium or format, as long as you give appropriate credit to the original author(s) and the source, provide a link to the Creative Commons licence, and indicate if changes were made. The images or other third party material in this article are included in the article's Creative Commons licence, unless indicated otherwise in a credit line to the material. If material is not included in the article's Creative Commons licence and your intended use is not permitted by statutory regulation or exceeds the permitted use, you will need to obtain permission directly from the copyright holder. To view a copy of this licence, visit http://creativecommons.org/licenses/by$\mathrm{nc} / 4.0 /$.

\section{REFERENCES}

1. Hunter SF. Overview and diagnosis of multiple sclerosis. Am J Manag Care. 2016;22:s141-50.

2. Cross AH, Naismith RT. Established and novel disease-modifying treatments in multiple sclerosis. J Intern Med. 2014;275:350-63.

3. Baum HM, Rothschild BB. The incidence and prevalence of reported multiple sclerosis. Ann Neurol. 1981;10:420-8.

4. Wallin MT, Culpepper WJ, Campbell JD, et al. The prevalence of MS in the United States: a populationbased estimate using health claims data. Neurology. 2019;92:e1029-40.

5. Lublin FD, Reingold SC, Cohen JA, et al. Defining the clinical course of multiple sclerosis: the 2013 revisions. Neurology. 2014;83:278-86.

6. Freedman MS, Selchen D, Prat A, Giacomini PS. Managing multiple sclerosis: treatment initiation, modification, and sequencing. Can J Neurol Sci. 2018;45:489-503.

7. Scalfari A, Neuhaus A, Degenhardt A, et al. The natural history of multiple sclerosis: a 
geographically based study 10: relapses and longterm disability. Brain. 2010;133:1914-29.

8. Costello K, Kalb R. The use of disease modifying therapies in multiple sclerosis: principles and current evidence. 2019. http://ms-coalition.org/wpcontent/uploads/2019/06/MSC_DMTPaper_ 062019.pdf. Accessed 26 Aug 2019.

9. Rae-Grant A, Day GS, Marrie RA, et al. Practice guideline recommendations summary: diseasemodifying therapies for adults with multiple sclerosis: report of the Guideline Development, Dissemination, and Implementation Subcommittee of the American Academy of Neurology. Neurology. 2018;90:777-88.

10. Grand'Maison F, Yeung M, Morrow SA, et al. Sequencing of high-efficacy disease-modifying therapies in multiple sclerosis: perspectives and approaches. Neural Regen Res. 2018;13:1871-4.

11. Pardo G, Jones DE. The sequence of disease-modifying therapies in relapsing multiple sclerosis: safety and immunologic considerations. J Neurol. 2017;264:2351-74.

12. Bonafede MM, Johnson BH, Wenten M, Watson C. Treatment patterns in disease-modifying therapy for patients with multiple sclerosis in the United States. Clin Ther. 2013;35:1501-12.

13. Nicholas J, Ko JJ, Park Y, et al. Assessment of treatment patterns associated with injectable diseasemodifying therapy among relapsing-remitting multiple sclerosis patients. Mult Scler J Exp Transl Clin. 2017;3:2055217317696114.
14. Salter AR, Marrie RA, Agashivala N, et al. Patient perspectives on switching disease-modifying therapies in the NARCOMS registry. Patient Prefer Adherence. 2014;8:971-9.

15. Giovannoni G, Marta M, Davis A, Turner B, Gnanapavan S, Schmierer K. Switching patients at high risk of PML from natalizumab to another disease-modifying therapy. Pract Neurol. 2016;16:389-93.

16. Deyo RA, Cherkin DC, Ciol MA. Adapting a clinical comorbidity index for use with ICD-9-CM administrative databases. J Clin Epidemiol. 1992;45:613-9.

17. Chastek BJ, Oleen-Burkey M, Lopez-Bresnahan MV. Medical chart validation of an algorithm for identifying multiple sclerosis relapse in healthcare claims. J Med Econ. 2010;13:618-25.

18. Sanchirico M, Caldwell-Tarr A, Mudumby P, Hashemi L, Dufour R. Treatment patterns, healthcare resource utilization, and costs among Medicare patients with multiple sclerosis in relation to disease-modifying therapy and corticosteroid treatment. Neurol Ther. 2019;8:121-33.

19. Degli Esposti L, Piccinni C, Sangiorgi D, et al. Changes in first-line injectable disease-modifying therapy for multiple sclerosis: predictors of nonadherence, switching, discontinuation, and interruption of drugs. Neurol Sci. 2017;38:589-94.

20. Braune S, Lang M, Bergmann A. Efficacy of fingolimod is superior to injectable disease modifying therapies in second-line therapy of relapsing remitting multiple sclerosis. J Neurol. 2016;263: 327-33. 